\title{
Efficient formation of adventitious shoots from immature zygotic embryos of the oilseed crop camelina
}

Rezaeva B.*, Kumlehn J.

Leibniz Institute of Plant Genetics and Crop Plant Research (IPK) Gatersleben, Plant Reproductive Biology, Gatersleben, Germany

*email: rezaeva@ipk-gatersleben.de

Camelina (Camelina sativa (L.) Crantz) is an oilseed plant that tolerates various adverse conditions, such as low temperature, drought, and nutrient-poor soils. In addition, the unique fatty acid profile of its seeds renders camelina particularly suitable for the provision of food and health products. While genetic engineering may greatly contribute to the improvement of yield and seed composition, it is dependent on efficient plant regeneration from cells that are accessible to DNA transfer, be it by employing Agrobacterium or by means of direct DNA delivery. In camelina, the current limits in plant regeneration constitute a bottleneck for the development and application of conventional as well as site-directed genetic engineering in elite germplasm. In the present investigation, immature zygotic embryos (IZEs) were dissected from camelina seeds of various developmental stages and used to induce adventitious shoot formation.

To this end, IZEs or parts thereof were cultivated under various conditions. On nutrient media containing either indole-3-acetic acid (IAA) or 6benzylaminopurine (BAP), leaf-like structures were formed at the surface of hypocotyls. Intriguingly, these structures emerged closer towards the root in the presence of BAP as compared to IAA. This response was the most intense when IZEs of 1.2 to $1.5 \mathrm{~mm}$ in length were cultivated as a whole rather than being divided in stem segments and cotyledons. Over time, the leaf-like structures converted into shoots which themselves gave rise to plantlets upon further cultivation. Current experiments are being conducted to determine the cellular origin of the shoots by histological examination. The information gained from this analysis will be essential for the implementation of the new regeneration system for genetic engineering approaches which are expected to substantially contribute to genetic improvements of camelina. 